\title{
Escape of high-energy oxygen ions through magnetopause reconnection under northward IMF
}

\author{
S. Kasahara ${ }^{1}$, H. Hasegawa ${ }^{1}$, K. Keika ${ }^{2}$, Y. Miyashita ${ }^{1}$, M. N. Nishino ${ }^{1}$, T. Sotirelis ${ }^{3}$, Y. Saito ${ }^{1}$, and T. Mukai ${ }^{1}$ \\ ${ }^{1}$ Institute of Space and Astronautical Science, JAXA, sagamihara, Kanagawa, Japan \\ ${ }^{2}$ Space Research Institute, Austrian Academy of Sciences, Schmiedlstrasse 6, Graz, Austria \\ ${ }^{3}$ Applied Physics Laboratory, 11100 Johns Hopkins Road Laurel, MD, USA
}

Received: 30 April 2008 - Revised: 10 October 2008 - Accepted: 29 October 2008 - Published: 5 December 2008

\begin{abstract}
During a storm recovery phase on 15 May 2005 , the Geotail spacecraft repeatedly observed highenergy $(>180 \mathrm{keV})$ oxygen ions in the dayside magnetosheath near the equatorial plane. We focused on the time period from 11:20 UT to 13:00 UT, when Geotail observed the oxygen ions and the interplanetary magnetic field (IMF) was constantly northward. The magnetic reconnection occurrence northward and duskward of Geotail is indicated by the Walén analysis and convective flows in the magnetopause boundary layer. Anisotropic pitch angle distributions of ions suggest that high-energy oxygen ions escaped from the northward of Geotail along the reconnected magnetic field lines. From the low-energy particle precipitation in the polar cap observed by DMSP, which is consistent with magnetic reconnection occurring between the magnetosheath field lines and the magnetospheric closed field lines, we conclude that these oxygen ions are of ring current origin. Our results thus suggest a new escape route of oxygen ions during northward IMF. In the present event, this escape mechanism is more dominant than the leakage via the finite Larmor radius effect across the dayside equatorial magnetopause.
\end{abstract}

Keywords. Magnetospheric physics (Magnetopause, cusp, and boundary layers; Magnetospheric configuration and dynamics; Storms and substorms)

\section{Introduction}

Escape of the Earth's oxygen ions $\left(\mathrm{O}^{+}\right.$and $\left.\mathrm{O}^{++}\right)$is a longstanding issue in magnetospheric physics. It is known that oxygen ions of low charge state are originated from the terrestrial ionosphere (cf. Abe et al., 1993; André and Yau, 1997; Nilsson et al., 2006) and that they follow various fates; for instance, some of them escape into the distant tail, and

Correspondence to: S. Kasahara

(kshr@stp.isas.jaxa.jp) some flow out across the duskside/dayside magnetopause (e.g. Seki et al., 2001; Ebihara et al., 2006).

Observations of energetic ions (including oxygen ions) in the magnetosheath have often been reported. Especially during geomagnetic storms, magnetospheric (ring current) ions can be the significant source, since they flow out from the magnetosphere (e.g. Daglis et al., 2003; Keika et al., 2005, and references therein). Furthermore, even in the upstream of the bow shock, energetic ions have been observed during geomagnetic disturbances, which are considered to be of magnetospheric origin (Winglee et al., 1996; Posner et al., 2002; Keika et al., 2004).

The flow out through the magnetopause plays a significant role in the ring current decay and the recovery of $D_{s t}$ index (Kozyra et al., 2002; Kozyra and Liemohn, 2003). The importance of the outflow is greater than or comparable to that of the charge exchange process during the storm main phase and the early recovery phase (Daglis et al., 2003; Keika et al., 2006). During strong magnetic storms, the energy density of oxygen ions often surpasses that of protons in the central $(L \sim 3-5)$ or relatively outer $(L \sim 5-7)$ ring current region (e.g. Hamilton et al., 1988; Daglis et al., 1997). Furthermore, McEntire et al. (1985) showed that the ratio of the integral fluxes (heavy ion flux)/(all ion flux) in the energy range of a few hundreds of $\mathrm{keV}$ became $\sim 1$ in the outer ring current region $(L \sim 9)$ at the dayside and the duskside. Understanding the loss process of energetic oxygen ions as well as other ion species through the magnetopause, therefore, is essential from the viewpoint of the storm time dynamics.

Possible candidates of the ion loss from the magnetosphere are (1) leakage due to the finite Larmor radius (FLR) effects and (2) escape along interconnected magnetospheremagnetosheath magnetic field lines that result from magnetic reconnection (e.g. Sibeck et al., 1987). Paschalidis et al. (1994) studied energetic ( $>50 \mathrm{keV})$ ions near the dayside equatorial magnetopause, by utilising the data obtained by AMPTE/CCE. They concluded that the leakage due to the

Published by Copernicus Publications on behalf of the European Geosciences Union. 
FLR effect is the dominant loss process. Recently, Marcucci et al. (2004) studied oxygen ion motion during a magnetopause skimming by Cluster, and found that a model based on the FLR effect can successfully reproduce the observation.

On the other hand, Zong et al. (2001) examined a storm event by using Geotail data, and found that oxygen ions $(>\sim 150 \mathrm{keV})$ escape through magnetic reconnection between the southward magnetosheath field and magnetospheric field lines near the equator. Although the escape via magnetic reconnection at a low latitude during southward Interplanetary magnetic field (IMF) has been reported (Speiser et al., 1981; Daly et al., 1984; Zong et al., 2001), there is little discussion on the escape via magnetic reconnection during northward IMF periods. One exception is the study by Onsager et al. (2001) who showed anisotropic phase space densities of ions (without mass discrimination) observed by the Polar spacecraft during northward IMF, and suggested that the ion escape was due to high-latitude reconnection with the closed magnetospheric field lines. Their observation, however, was limited to ions with energy $<20 \mathrm{keV} / \mathrm{q}$, and could not mention the ion species due to the lack of a mass analyser.

In this paper, we present for the first time evidence of the escape of high-energy ( $>180 \mathrm{keV}$ ) oxygen ions along magnetic field lines produced by magnetic reconnection under northward IMF.

\section{Instrumentation}

The Ion Composition System (ICS) sensor is a part of the Energetic Particles and Ion Composition (EPIC) instrument onboard Geotail (Williams et al., 1994). ICS consists of three individual sensor heads; two of them are identical and measure ion spectra and compositions, while the third head measures electron spectra.

The ion detectors sweep out electrons with energy $<200 \mathrm{keV}$ by a magnet. Their heads are set on the northern and southern sides of the satellite body, and the northern (southern) aperture is centred $23^{\circ}$ above (below) the spin plane, with $30^{\circ}$ conical Field of View. Energy of ions is measured by $350 \mu \mathrm{m}$ silicon Solid State Detectors (SSDs). Timeof-Flight system with start/stop MicroChannel Plate (MCP) assemblies, in combination with an SSD assembly, provides information on the mass of each incoming ion. Measurement energies are $187-3565 \mathrm{keV}$ for heavy $(\mathrm{C}, \mathrm{N}$, and $\mathrm{O})$ ions and $58-3005 \mathrm{keV}$ for protons. Heavy ions (atomic numbers of 12-16) are regarded as oxygen ions in this analysis. Pitch Angle (PA) distributions obtained over $180^{\circ}$ are divided into nine angular bins. Although these sensor heads cannot cover $180^{\circ}$ fan, most of the whole PA range is scanned in combination with the spacecraft spin motion every $\sim 3 \mathrm{~s}$ (spin period). The azimuthal angle in the spin plane is divided into $16 \mathrm{sec}-$ tors.
We also use the magnetic field and the low-energy particle data, which are respectively obtained by the Magnetic Field sensor (MGF) (Kokubun, 1994) and the Low Energy Particle detector (LEP) onboard Geotail (Mukai et al., 1994). LEPEAi (for ions) and LEP-EAe (electrons) consist of spherical electrostatic analysers and MCPs or Channel Electron Multipliers (CEMs). Measurement energies during the present event are $\sim 0.1-40 \mathrm{keV} / \mathrm{q}$.

Precipitation of low-energy ions and electrons is observed at a low altitude $(\sim 850 \mathrm{~km})$ by the Precipitating Electron and Ion Spectrometer onboard DMSP (Hardy et al., 1984). The instrument looks toward the satellite zenith, and the energy coverage is $0.03-30 \mathrm{keV} / \mathrm{q}$. The convective flow is measured by retarding potential analysers and ion driftmeters (Greenspan et al., 1986; see also Hanson et al., 1981; Heelis et al., 1981, for the measurement principles). DMSP is in a sun-synchronous orbit with the orbital period of $101 \mathrm{~min}$.

In order to monitor the solar wind, we use the data obtained by the Solar Wind Electron, Proton, and Alpha Monitor (McComas et al., 1998) and the Magnetometer instrument (Smith et al., 1998) onboard the ACE spacecraft.

\section{Observations}

\subsection{Solar wind and magnetospheric conditions}

On 15 May 2005, a strong geomagnetic storm occurred, with the minimum Sym- $H$ index of $-305 \mathrm{nT}$ at 08:21 UT. Figure 1 shows solar wind moment data and IMF, observed by the ACE satellite, which was located $\sim 250 R_{E}$ upstream of the Earth. The data are shifted by $34 \mathrm{~min}$ forward, in order to see their effects on the Earth's magnetosphere. The interplanetary shock in front of a coronal mass ejection (CME) would have arrived at $\sim 02: 40 \mathrm{UT}$, as can be seen in the steplike increase of the magnetic field intensity $\left(B_{t}\right)$. The proton density $(\mathrm{Np})$, velocity $\left(V_{x}\right)$, and thus dynamic pressure $\left(P_{d}\right)$ tremendously increased downstream of the shock. The main body of the CME arrived at $\sim 06: 00 \mathrm{UT}$. $\left|V_{x}\right|$ exceeded $800 \mathrm{~km} / \mathrm{s}$, with relatively large $\left|V_{y}\right|$ and $\left|V_{z}\right|(\sim 100 \mathrm{~km} / \mathrm{s})$. Inside the CME, clear signatures of a magnetic flux rope are seen in the magnetic field data; after 06:00 UT, $B_{x}$ was constantly negative, while the magnetic field rotated in the $y-z$ plane.

The storm initiation roughly coincided with the southward turning of the IMF at $\sim 06: 00 \mathrm{UT}$. After $3 \mathrm{~h}$ of the southward IMF period, it turned back northward at 09:00 UT, and was continuously northward until $\sim$ 18:00 UT. Sym- $H$ index is also displayed at the bottom in Fig. 1. A rapid recovery phase continued until $~ 18: 00$ UT on 15 May, followed by a slow recovery phase until 20 May (not shown).

\subsection{Geotail observations}

Figure 2 displays the omni-directional energy-time spectrograms of electrons and ions, the ion moments calculated from 


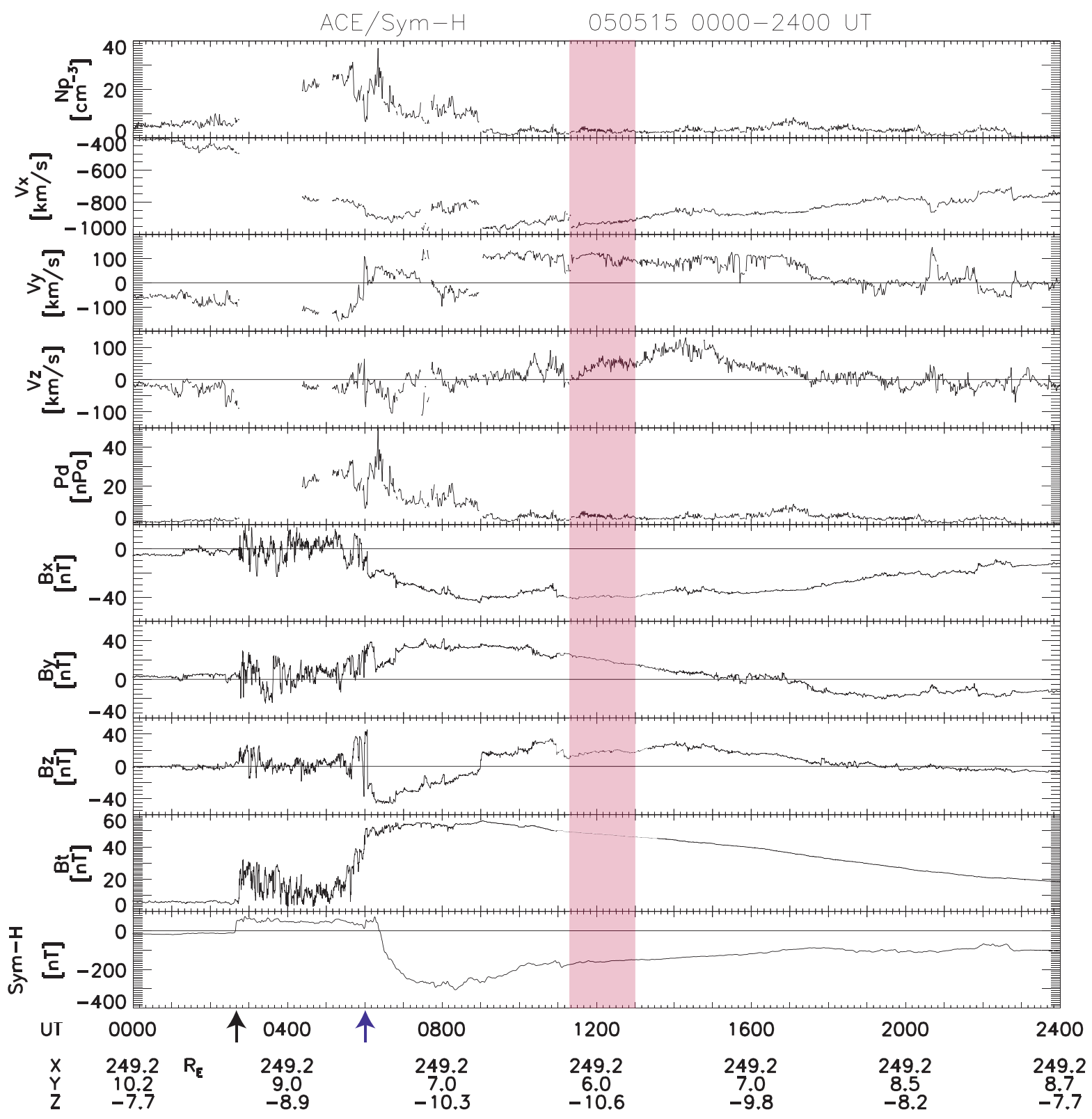

Fig. 1. Solar wind data (in GSM) obtained by ACE (shifted 34 min forward). The Sym- $H$ index is also displayed at the bottom. The hatched period is examined by using Geotail data. The arrivals of the interplanetary shock front and the main body of the CME are seen at 02:40 UT (black arrow) and 06:00 UT (purple arrow), respectively.

the LEP data, and the magnetic field in GSM, from 11:20 to 13:00 UT. This time period is during the rapid recovery phase (Sym- $H \sim-150 \mathrm{nT}$ and increasing). Geotail was located in the dayside magnetosheath or the magnetopause boundary layer. The location of Geotail is also displayed at the bottom of Fig. 2. The bar on the top of Fig. 2 roughly indicates the region in which Geotail was located; the light blue, yellow, and maroon mean the magnetosphere $(\mathrm{M})$, the magnetosheath (Sh), and the boundary layer (B), respectively. In the energy-time spectrograms (top two panels), sporadic enhancements of medium-energy particle fluxes $(\sim 1 \mathrm{keV}$ for electrons and $\sim 10 \mathrm{keV} / \mathrm{q}$ for ions) are seen (e.g. 11:50 UT and 12:22 UT) during magnetosheath periods.

For moment calculation, all ions are assumed to be protons, since the LEP-EAi instrument does not discriminate ion mass. Thinner magenta lines in the $V_{x}, V_{y}$, and $V_{z}$ panels illustrate three components of the velocity perpendicular to the ambient magnetic field. The magnetic field 

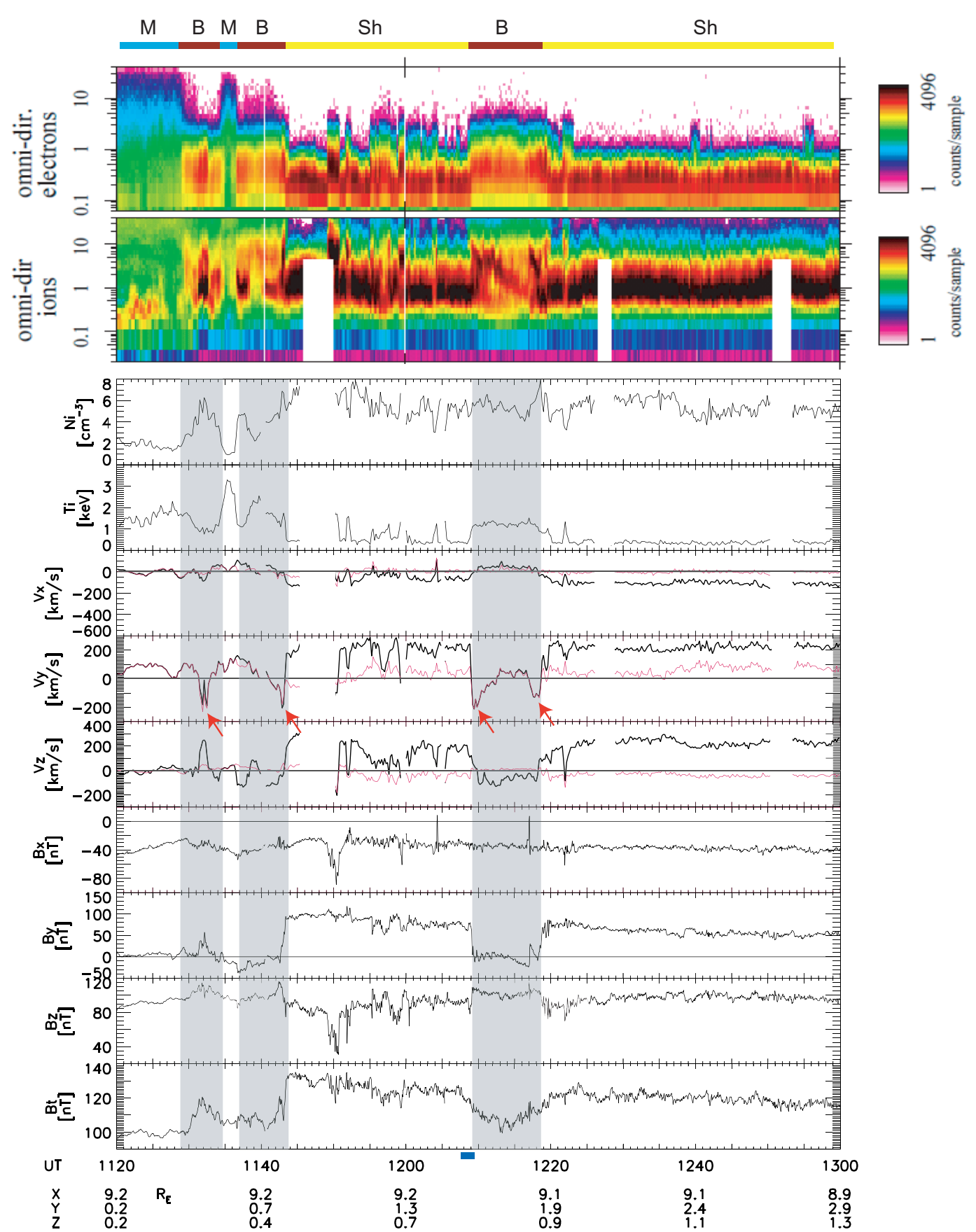

Fig. 2. E-t spectrograms of electrons (top panel) and of ions (second panel), ion moments calculated from LEP data, and the magnetic field. The scales are logarithmic for energy (ordinate) and count rates (colour). Periods of the magnetopause boundary layer are also hatched. At the outer edge of the layer, dawnward flows $\left(V_{y}<0\right)$ were observed (indicated by red arrows). The satellite position is described in GSM coordinates. Thinner magenta lines in the $V_{x}, V_{y}$, and $V_{z}$ panels are the components of the velocity perpendicular to the ambient magnetic field. The bar on the top roughly indicates the region in which Geotail was located; the light blue, yellow, and maroon mean the magnetosphere (M), the magnetosheath $(\mathrm{Sh})$, and the boundary layer (B), respectively.

on the magnetosheath side is strongly northward with large duskward and tailward components: $\boldsymbol{B} \sim(-40,100,90) \mathrm{nT}$. The bulk flow of solar wind in the magnetosheath is nearly parallel to the magnetic field: $\boldsymbol{V} \sim(-100,200,200) \mathrm{km} / \mathrm{s}$. The hatched (grey) periods are the magnetopause boundary layer; the density and temperature are higher and lower, 

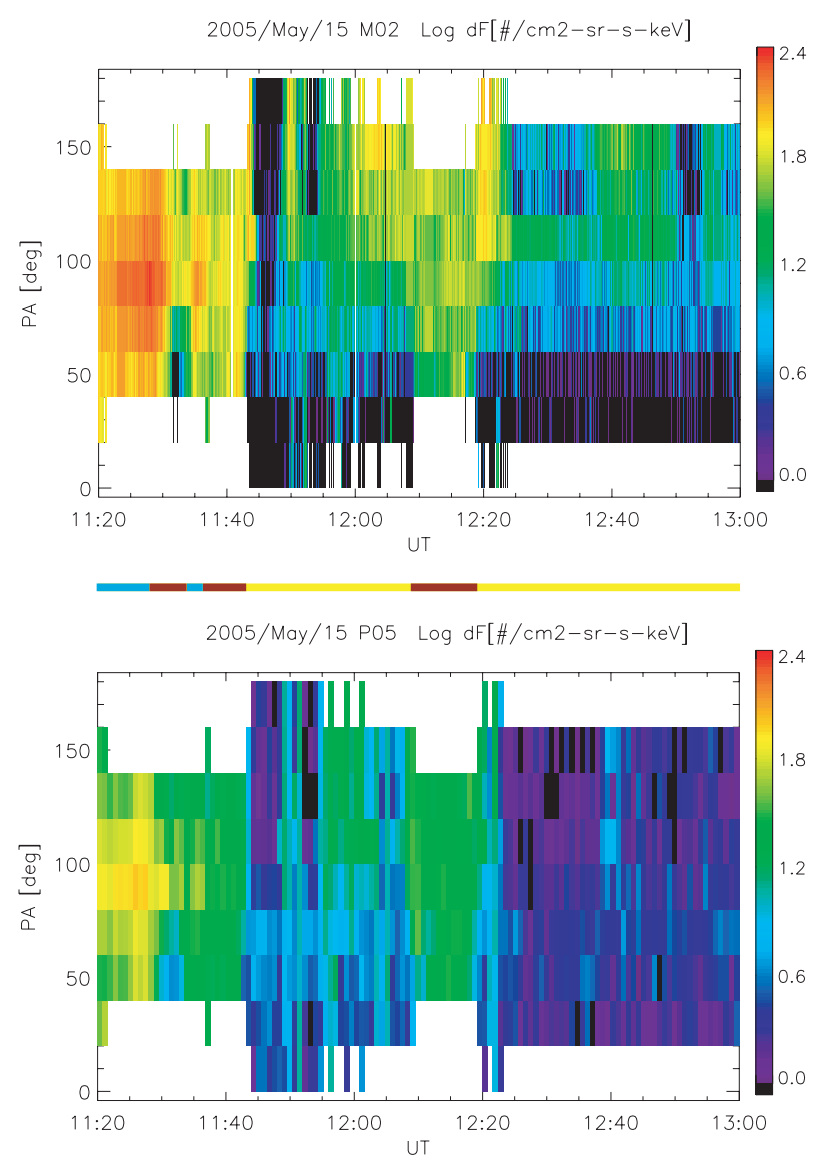

Fig. 3. Pitch angle distributions of oxygen ions (187-221 keV) in the upper panel and protons $(154-228 \mathrm{keV})$ in the lower. The bar in the middle is identical to the one in Fig. 2.

respectively, by a factor of two than those in the magnetosphere. The anomalously strong magnetic field strength in the magnetosheath exceeds the magnetic field strength inside the magnetosphere.

Figure 3 shows PA distributions of energetic ions from 11:20 to 13:00 UT. The upper panel is for high-energy (187$221 \mathrm{keV}$ ) oxygen ions. The lower panel shows high-energy (154-228 keV) protons. The bar in the middle of Fig. 3 is identical to the one in Fig. 2. Geotail observed a significant flux of high-energy oxygen ions, which surpasses the proton flux. The flows of oxygen ions antiparallel to the magnetic field are seen at $\sim 11: 44$ UT, 11:48-11:52 UT, 11:54-12:09 UT, and 12:19-13:00 UT. Furthermore, during 11:48-11:52 UT and 12:01-12:06 UT, the fluxes at PA $\sim 150^{\circ}$ are much more intense than those at $\mathrm{PA} \sim 100^{\circ}$. These flows of energetic oxygen ions are the most striking feature in this event, and their nature is the subject of this paper. Periods straddling the magnetosheath and the boundary layer $(\sim 11: 44 \mathrm{UT}, \sim 12: 08 \mathrm{UT}$, and $\sim 12: 19 \mathrm{UT})$ are discussed later, since they provide essential information on the magnetic field topology. The schematic showing our interpretations is found in Fig. 4.

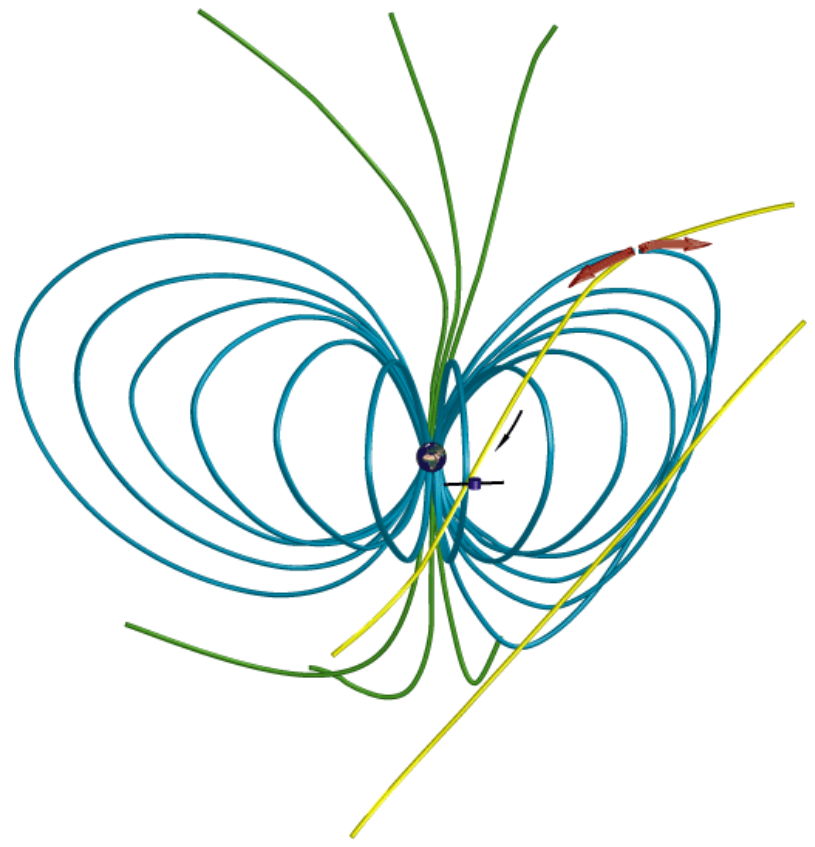

Fig. 4. Schematic diagram of the magnetic configuration and the location of Geotail (at the dayside equatorial plane). Green, blue, and yellow lines indicate open, closed, and interplanetary magnetic field lines, respectively. Maroon arrows display reconnection jets (field line motions), while a black arrow shows the anti-field aligned flow of energetic ions observed by Geotail.

In the boundary layer, the remarkable negative $V_{y}$ (i.e. dawnward flows of the order of $100 \mathrm{~km} / \mathrm{s}$ represented by red arrows in Fig. 2) are almost equal to the y-component of the velocity perpendicular to the background magnetic field; this is oppositely directed to the solar wind flow in the magnetosheath, and hence it is suggested that the observed flows are reconnection jets (cf. Gosling et al., 1990). The dawnward jet is consistent with the occurrence of magnetic reconnection duskward of the satellite location. In addition, the rather small IMF clock angle $\left(B_{y} \sim B_{z}\right)$ implies that the reconnection site was at a high latitude, although we do not rule out the possibility of component reconnection at a low latitude (but northward of Geotail).

It should be noted that the flux increases at medium energy in the magnetosheath side (see top two panels of Fig. 2) coincide with the high-energy ion flows in Fig. 3. The anisotropy of the medium-energy ion distribution functions (although not shown) is consistent with that expected for escaping ring current ions and/or reflected magnetosheath ions (Cowley, 1982, 1995); it suggests that the satellite was on the reconnected flux tube.

We performed the Walén analysis (cf. Sonnerup et al., 1987) to confirm the reconnection occurrence and its location. The interval used for the analysis is 12:07:3712:09:25 UT, as indicated by the blue bar at the bottom of Fig. 2. For the calculation, we assumed the isotropy of 


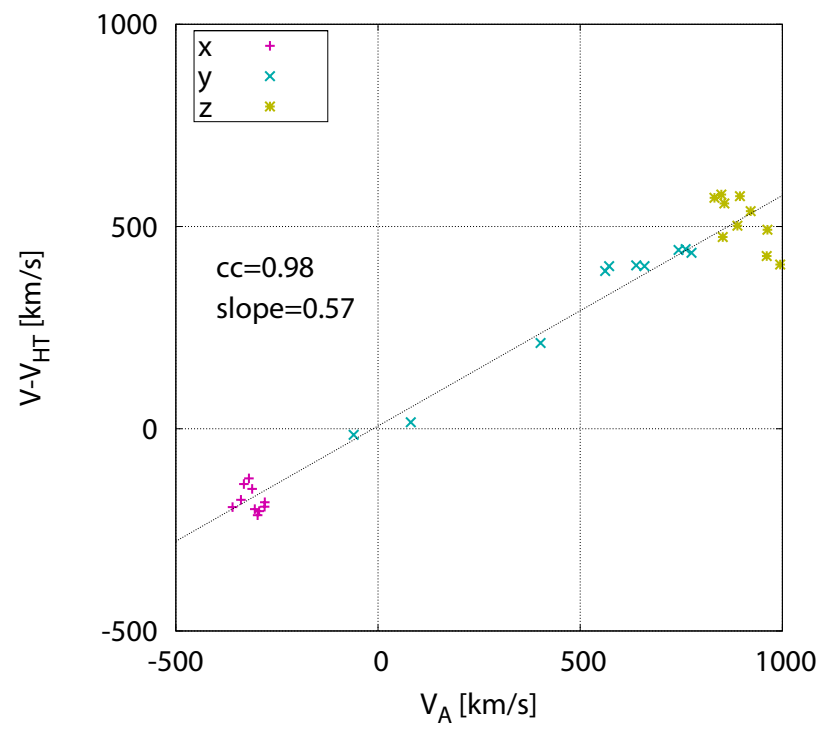

Fig. 5. Walén plot in GSM for the interval of 12:07:3712:09:25 UT. Black, red, and green represent the $\mathrm{x}, \mathrm{y}$, and $\mathrm{z}$ components, respectively. The velocity of the deHoffman-Teller frame in GSM was found to be $(124,-199,-368) \mathrm{km} / \mathrm{s}$ with the correlation coefficient of 0.98 .

the pressure and that all detected ions were protons. The deHoffman-Teller (dHT) frame was determined well with the correlation coefficient between components of $-\boldsymbol{V} \times \boldsymbol{B}$ and of $-\boldsymbol{V}_{H T} \times \boldsymbol{B}$ of 0.98 , where $\boldsymbol{V}$ and $\boldsymbol{V}_{H T}$ are the velocities measured in the spacecraft-rest frame. The resultant dHT velocity is $\boldsymbol{V}_{H T}=(124,-199,-368) \mathrm{km} / \mathrm{s}$ in GSM; its southward and dawnward components are consistent with the reconnection occurrence northward and duskward of Geotail. Figure 5 shows that flow velocities in the dHT frame have a linear correlation with the local Alfvén velocities. The correlation coefficient is 0.98 . The positive slope indicates that the normal component of the magnetic field at the rotational discontinuity magnetopause was earthward (e.g. Sonnerup et al., 1981). These are further evidence of the reconnection occurrence and are consistent with its location being northward and duskward of Geotail under the northward and duskward IMF condition (Fig. 4). We discuss the reason of a relatively small slope $(\sim 0.57)$ later.

\section{Precipitation in the Polar region}

Although magnetic reconnection northward and duskward of Geotail is indicated by the above analysis, we have two candidates on the reconnection type: one is reconnection with the equatorward-of-cusp (closed) magnetospheric field, and the other is with the poleward-of-cusp (open) field (i.e. lobe reconnection). In order to confirm that at least the former type of reconnection have occurred, we analyse the particle precipitation observed by DMSP at a low altitude $(\sim 850 \mathrm{~km})$.

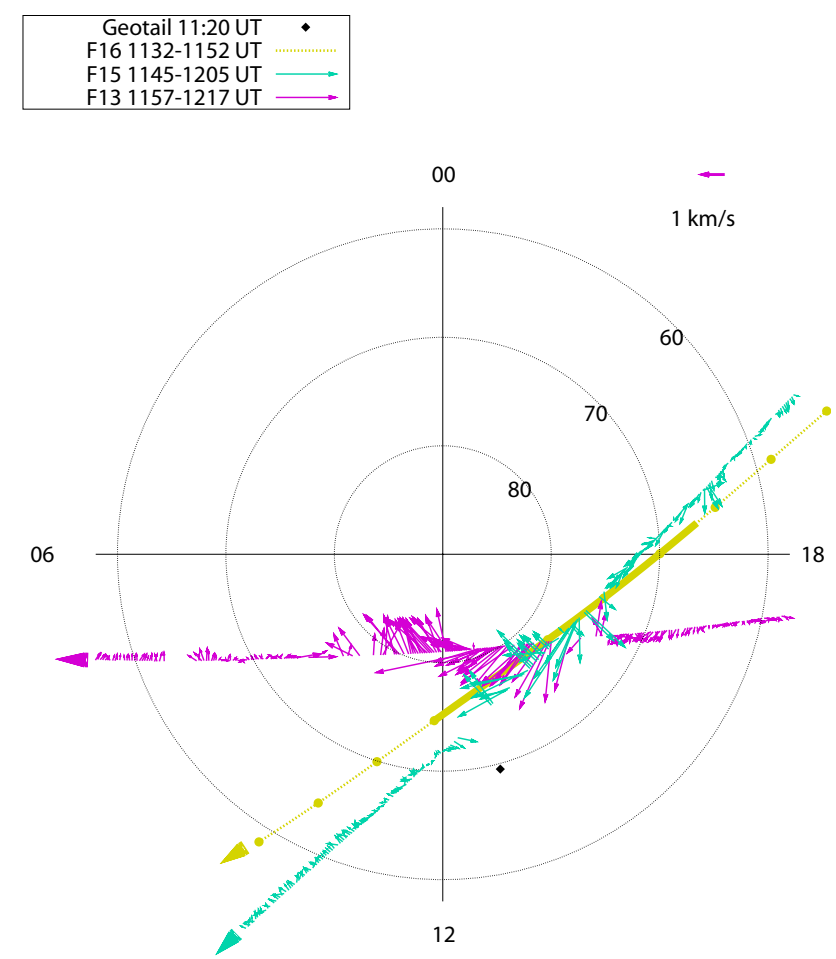

Fig. 6. Orbits of the DMSP satellites and observed flows. They moved from the dusk sector to the morning sector. Magnetic local time and latitude are displayed. The top (bottom) is the midnight (noon), and the left (right) is the dawn (dusk). The thick solid line in the F16 track indicates the polar cap region derived from the precipitation signature. The mapped position of Geotail at 11:20 UT is also shown.

Figure 6 shows the orbits of the DMSP satellites (F13, F15, and F16), which passed through the dayside sector in the Northern Hemisphere. The plot is for the northward IMF period that is covered by the Geotail observation (11:32-11:52 UT for F16, 11:45-12:05 UT for F15, and 11:57-12:17 UT for F13). Observed horizontal flows are superposed (except for F16, which does not provide flow data). The thick solid line for F16 indicates the polar cap period, which is determined by the particle data shown below. The Geotail footpoint at 11:20 UT, when it was located inside the magnetosphere, is also shown as a rough guide. The Geotail position is mapped by a model magnetic field (Tsyganenko and Stern, 1996) using the averaged solar wind parameters obtained by ACE and $1 \mathrm{~h}$ average of Sym- $H$ index (instead of $D_{s t}$ ).

Apparent feature in Fig. 6 is strong sunward flows observed by F13 and F15 at $\sim 14-16.5$ MLT. Furthermore, in a closer look, one would notice that F15 also observed abrupt changes of the flow direction; in the evening/post-noon sector, both the sunward flow and the anti-sunward/dawnward flow are observed by F15. This indicates that the convection pattern was not steady in this period, and thus the reconnection occurrence would not have been stable. 


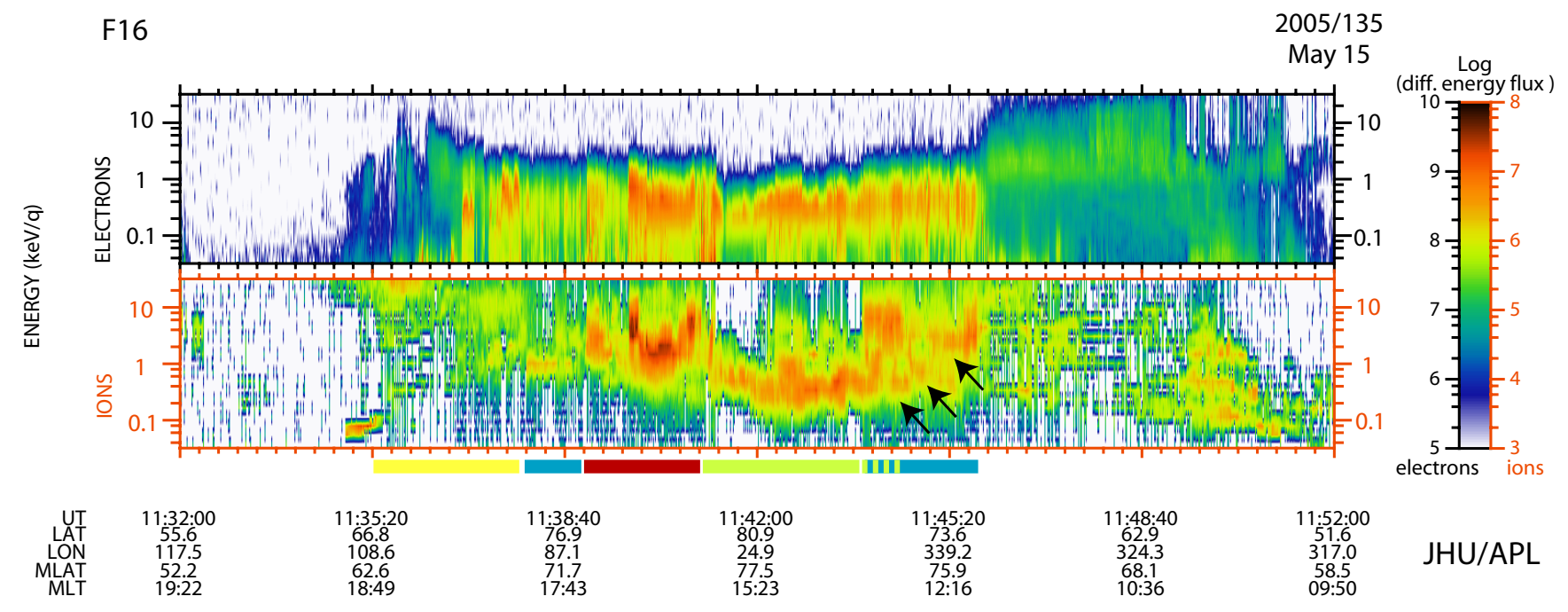

Fig. 7. Energy-time spectrograms of electrons and ions obtained by the DMSP F16 satellite from 11:32 to 11:52 UT. The scales are logarithmic for ordinate and colour (differential energy flux). The colour bars at the bottom correspond to precipitation regions shown in Fig. 9.

Figure 7 is the precipitating particle data observed by F16 during the northward IMF period. The energy-time spectrograms for electrons (top) and ions (bottom) are displayed. In the lower-latitude region (before $\sim 11: 36: 40 \mathrm{UT}$ and after 11:46:00 UT), medium-energy electrons $(>1 \mathrm{keV})$ and ions $(>10 \mathrm{keV} / \mathrm{q})$ are seen. The higher peak energies and the lower counts of low-energy particles evidence that these populations are central plasmasheet/ring current particles trapped by the closed field.

In the polar cap period ( 11:36:40-11:46:00 UT), significant precipitation of the low-energy particles is seen. The average energy of electrons $(\sim 200 \mathrm{eV})$ is comparable to those in the magnetosheath (see Fig. 2). Ions with $1-10 \mathrm{keV} / \mathrm{q}$ energies in the polar cap region can be interpreted as having been accelerated at/near the X-line. From 11:39 to 11:41 UT, such accelerated particles are seen, and the precipitation is categorised as of cusp (Newell et al., 1991a, b). This cusp, which is separate from the open/closed boundary, suggests the occurrence of lobe reconnection (e.g. Pitout et al., 2002). Furthermore, the complex structure in the spectrogram for this period may be due to transient occurrence of lobe reconnection, which is consistent with abrupt changes of the convection pattern observed by F15.

On the other hand, precipitation in the low-latitude boundary layer-like (LLBL-like) region (Newell et al., 1991b) during 11:44:20-11:45:50 UT shows another feature. As indicated by black arrows, the energy cutoffs show step-like increase with decreasing latitude. This is a well-known manifestation of pulse mode reconnection between the closed field line and the magnetosheath field line: the step-like signature is attributed to a sequence of the flux tubes moving poleward, which started at the reconnection site along the dayside open/closed boundary (see Farrugia et al., 1998).
Mantle type precipitation during 11:41-11:44 UT shows energy decrease and increase, associated respectively with increase and decrease of magnetic latitude. The minimum energy cutoff is roughly coincident with the peak latitude at $\sim 11: 43$ UT. The origin of this energy-dispersed feature is discussed later.

After 11:43:50 UT, coexistence of two components, i.e. the mantle type low-energy component and the LLBL type accelerated component are seen simultaneously: this can be attributed to the ion drift motion across the flux tube. LLBL ions $(\sim 1-10 \mathrm{keV})$ can transfer to the mantle flux tube via the curvature/gradient drift. The coexistence may also be explained by other mechanisms such as lobe reconnection in the Southern Hemisphere. However, providing definitive explanation is beyond the scope of this paper.

Figure 8 displays the energy-time spectrograms obtained by F13 with the horizontal flow in the noon-midnight direction at the top. The time period is 11:57-12:17 UT, 25 min later than the F16 pass. The bottom panel shows a clear signature of ion energy dispersion from 12:02:40 to 12:04:00 UT. The initiation of the dispersion signature is roughly coincident with the beginning of the strong sunward flow, which indicates lobe reconnection in the evening/postnoon sector. This observation is consistent with the cusp-type precipitation seen by F16 in the same MLT and latitudinal region, explained by lobe reconnection.

The other point in this plot is the existence of accelerated ions at the duskside mantle/LLBL (12:08:40-12:11:40 UT). These ions are observed with the anti-sunward convection near the duskside open/closed boundary (observed at $\sim 12: 11: 40$ UT). These accelerated ions cannot be explained by the transport from the cusp in the evening sector indicated above; even if the lobe cell extends to the dawnside, ions with 


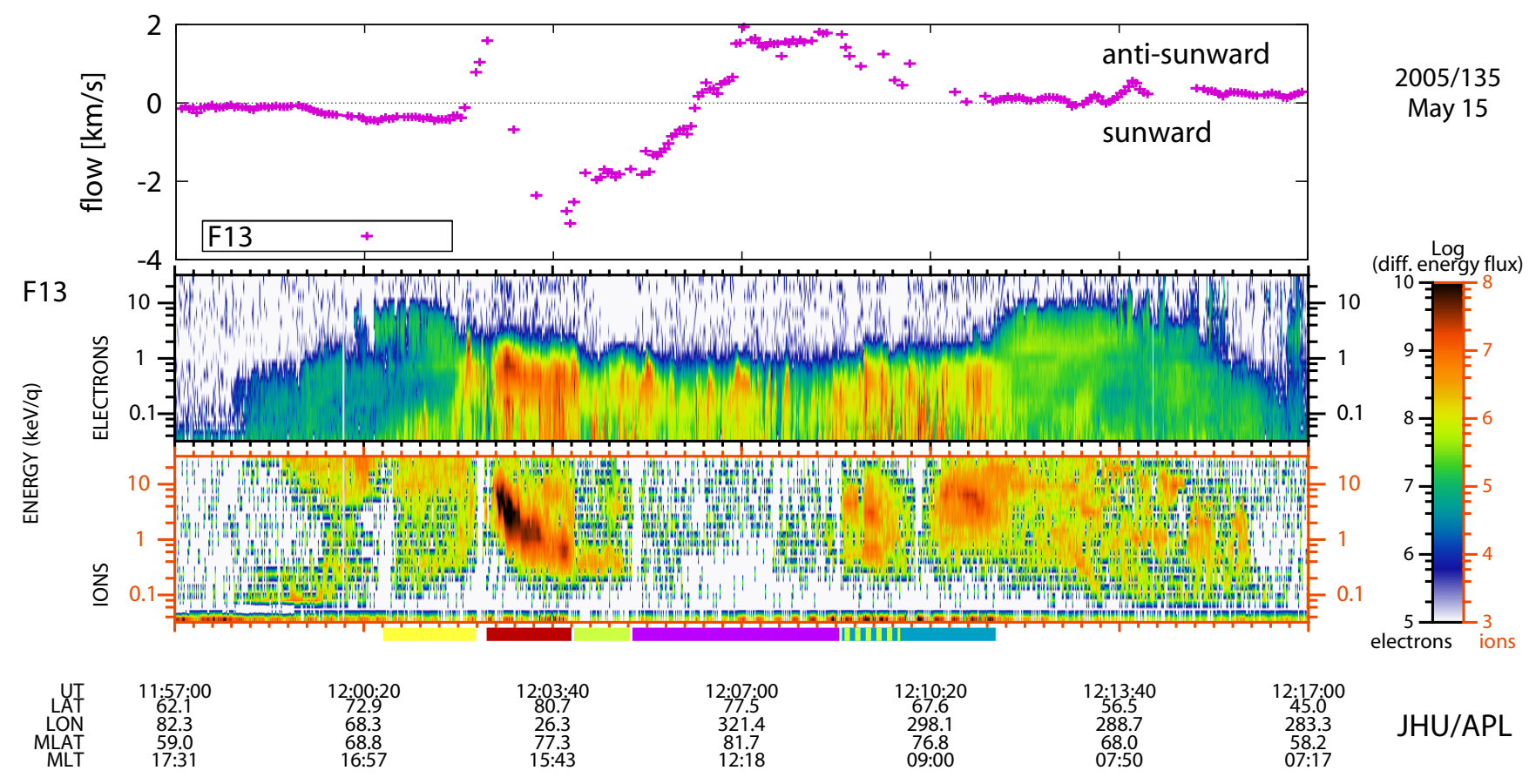

Fig. 8. Sunward/anti-sunward flow (top) and energy-time spectrograms (bottom two panels) of electrons and ions obtained by the DMSP F13 satellite from 11:57 to 12:17 UT. The meaning of the bottom colour bars is the same as in Fig. 9.

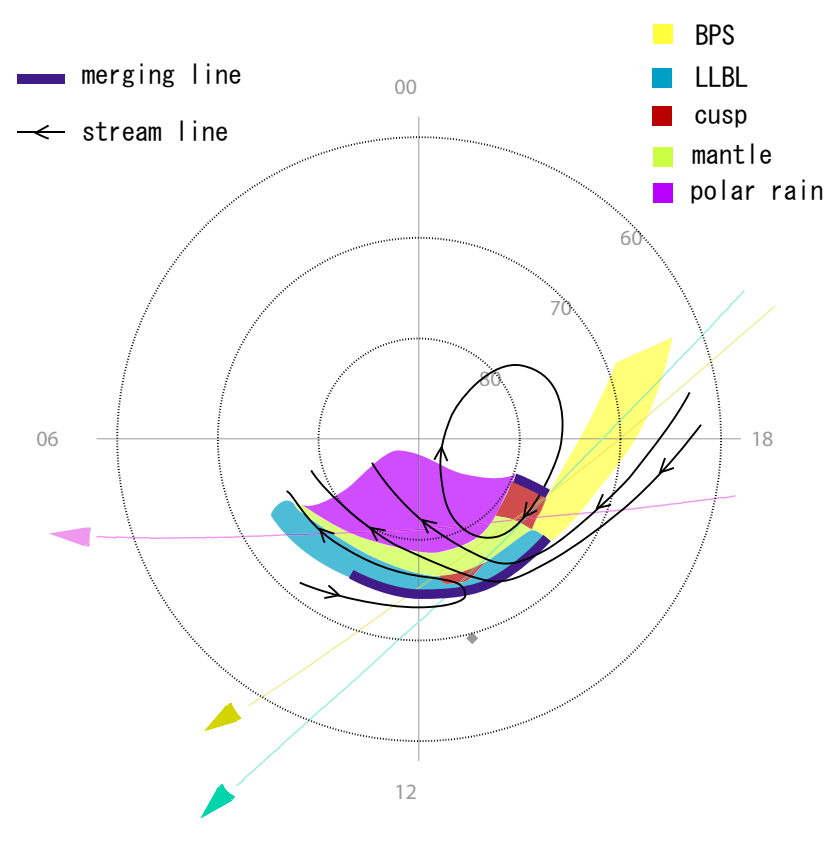

Fig. 9. Schematic diagram illustrating reconnection sites and precipitation signatures. DMSP orbits are also displayed (F13 in magenta, F15 cyan, and F16 yellow).

such energy would have precipitated before the flux tube is convected to the duskside (see the rapid energy decrease in the cusp in Fig. 8). It seems to be more likely that accelerated ions in this period are associated with the merging line along the dayside open/closed boundary suggested above.

Figure 9 illustrates our interpretation of the DMSP data. As indicated above, transient reconnection would have occurred in the focused period, hence it is impossible to make one schematic diagram which is completely consistent with all data. Nevertheless, Fig. 9 shows most of important aspects for our purpose.

F13 passed the polar cap region in the order of boundary plasma sheet (BPS), cusp, mantle, polar rain region, and mantle/LLBL (see also Fig. 8; cf. Newell et al., 1991b). Energy-time spectrograms of F15 also show consistent signatures with this schematic diagram, although they are not shown here because the data include unrealistic (instrumental) energy cutoff. F16 also observed the high-latitude cusp, mantle, and LLBL in this order (see Fig. 7). As pointed out above, F16 mantle precipitation ( 11:41-11:44 UT) shows remarkable energy decrease/increase along the track. This variation can be interpreted in terms of the time-of-flight effect. Since higher-energy (i.e. faster) ions precipitate earlier, the energy cutoff can correspond to the distance from the low-latitude merging line on the open/closed boundary to the satellite track along the convection stream line. Therefore, the lowest energy cutoff is detected at the farthest point from the open/closed boundary.

Note that it is unlikely that this mantle associates with the high-latitude cusp, since the low-energy cutoff does not smoothly connect to the cusp precipitation signature at 
$\sim$ 11:41:20 UT (Fig. 7). The electron spectrum also shows abrupt change at the same time. It should be also noted that the average slope of the step-like increase of the ion cutoff energy in the LLBL well matches the smooth slope in the mantle region. These features are further evidence that the observed mantle is associated with reconnection occurring on the open/closed boundary, not at the high-latitude lobe magnetopause.

In summary, the DMSP data indicates the reconnection occurrence at two sites quasi-simultaneously: one is the highlatitude evening/post-noon sector, and the other is the dayside open/closed boundary. This result supports our interpretation in Fig. 4.

\section{Discussion}

\subsection{Escape through reconnection during northward IMF}

Magnetic field configurations and the route of the oxygen ion escape inferred from the above observations are illustrated in Fig. 4. Note that we do not rule out the occurrence of component reconnection near the subsolar point northward of Geotail, although it is not shown in Fig. 4. Although the most likely reconnection site is the dusk high-latitude magnetopause, component reconnection near the subsolar point is possible since the IMF $B_{y}$ is comparable to $B_{z}$ (cf. Moore et al., 2002).

The signatures of energetic oxygen ion escape via reconnection northward of Geotail were simultaneously observed at $\sim 11: 44-11: 45$ UT: (1) the energetic oxygen ion outflow in Fig. 3, (2) the reversal of $V_{y}$ across the magnetopause in Fig. 2, which suggests magnetic reconnection (cf. Gosling et al., 1990), and (3)accelerated ions in Fig. 7, which suggests that the magnetosheath field reconnected with the closed field lines. Similar features are found in other periods, although simultaneous observation is not obtained due to a poor coincidence with the DMSP observation. The Walén analysis for 12:07:37-12:09:25 UT further evidenced the occurrence of reconnection duskward and northward of Geotail. The Walén test for 11:43-11:44 UT does not clearly show the evidence of reconnection; it may suggest that the magnetopause was not a rotational discontinuity at the Geotail location during that time interval. However, the above observations (reversal of $V_{y}$ and precipitation at the low altitude) show the occurrence of magnetic reconnection at $\sim 11: 44$ UT somewhere on the magnetopause equatorward of the northern cusp. Oxygen ion detection at 11:44 UT despite such a location (i.e. locally not being a reconnection site) is probably due to a large Larmor radius, as discussed below.

In Fig. 3, the oxygen flux is more intense in the direction of PA $>90^{\circ}$. This anisotropy is consistent with the escape along reconnected field lines generated at the reconnection site northward of Geotail. High-energy oxygen ions were observed for a much longer time period (after 12:20 UT), com- pared to high-energy protons, probably due to their larger gyroradii; particles with larger gyroradii have a higher probability of being observed if a spacecraft is at a certain distance from the reconnected flux tube.

Outside the magnetopause boundary layer $(\sim 11: 44 \mathrm{UT}$, 12:01-12:06 UT, and 12:19-12:22 UT), the strongest anisotropy of energetic ions was observed. These do not appear to be the ions leaked across the low-latitude magnetopause via the FLR effects, since the leakage due to the FLR cannot explain the observed anisotropy. In addition, the fluxes at $\mathrm{PA} \sim 150^{\circ}$ are much more intense than those at $\mathrm{PA} \sim 100^{\circ}$ for some flows. Thus, we conclude that the escape through magnetic reconnection during the northward IMF is dominant on the dayside in this event, although we do not rule out the significance of the leakage via the FLR effects on the duskside.

\subsection{Small slope of the Walén test}

It is interesting to note the relatively small slope $(\sim 0.57)$ in the Walén analysis (see Fig. 5). If the plasma pressure in the magnetosheath is isotropic and all ions are protons, the slope should be unity. One possibility of the small slope is anisotropy of the pressure $\left(P_{\|}-P_{\perp} \neq 0\right)$, since the predicted velocity is modified by being multiplied by $\sqrt{1-\mu_{0}\left(P_{\|}-P_{\perp}\right) / B^{2}}$, where $B$ is the magnetic field strength (cf. Sonnerup et al., 1995). The order of anisotropy can be estimated as

$\left(P_{\|}-P_{\perp}\right) / B^{2} \sim P / B^{2}$,

where $P$ is the pressure for an isotropic plasma. It is calculated by using $n=5 \mathrm{cc}^{-1}, T=0.5 \mathrm{keV}$, and $B=100 \mathrm{nT}$ (12:07-12:09 UT, see Fig. 2) to obtain $\mu_{0} P / B^{2} \sim 0.03 \ll 1$, and thus $\sqrt{1-\mu_{0}\left(P_{\|}-P_{\perp}\right) / B^{2}} \sim 1$. Therefore, the anisotropy cannot explain the small slope in Fig. 5. It should be noted, however, that such a small $\mu_{0} P / B^{2}$ (small plasma beta) can have a significant influence on the reconnection occurrence (Paschmann et al., 1986, and references therein).

Another possible explanation of the small slope is the existence of thermal oxygen ions; the above Alfvén velocity was calculated with the assumption that all ions were protons, hence it tends to be overestimated. If we assume that oxygen ions contribute to $15 \%$ of total number density, the slope is modified to 0.98 . Although the frequency of plasma oscillation (not shown here) suggests that the plasma density used for the calculation of Alfvén velocity (obtained by LEP) is slightly underestimated, the above value (oxygen ion contribution of $15 \%$ ) is reduced only by a factor of $\sim 2-3$.

\subsection{Outflow rates and the contribution to $D_{s t}$ recovery}

Finally, we estimate the oxygen ion outflow rate and its possible effect on the $D_{s t}$ recovery (the results are summarised in Table 1). 
Table 1. Summary of Oxygen ion loss rate and the effect on $D_{s t}$ (normalised by the unit escape area of $1 R_{E}^{2}$ ).

\begin{tabular}{lcc}
\hline Number flux & $\begin{array}{c}\text { Escape rate } \\
\left.\text { (particles } / \mathrm{s} / R_{E}{ }^{2}\right)\end{array}$ & $\begin{array}{c}\left(d D_{s t} / d t\right)_{\text {o.e. }} \\
\left(\mathrm{nT} / \mathrm{day} / R_{E}{ }^{2}\right)\end{array}$ \\
\hline$F(187-3565 \mathrm{keV})$ & $3 \times 10^{21}$ & 0.04 \\
$F^{\prime}$ (calculated from moments) & $6 \times 10^{23}$ & 11 \\
\hline
\end{tabular}

The outflow rate $\lambda$ can be calculated by the equation

$\lambda=\int d E \int d \Omega \int \boldsymbol{J} \cdot d \boldsymbol{S}$,

where $\boldsymbol{J}$ indicates the differential number flux of oxygen ions (as a function of the energy and pitch angle in the unit of $\left.\mathrm{cm}^{-2} \mathrm{sr}^{-1} \mathrm{~s}^{-1} \mathrm{keV}^{-1}\right), \int d \boldsymbol{S}$ means the integral over the area in which reconnected field lines are present. The direction of the vector $d \boldsymbol{S}$ is anti-parallel to the magnetosheath field lines in front of the magnetopause. $E$ and $\Omega$ indicate energy and solid angle, respectively. We assume homogeneity of $\boldsymbol{J}$ over the integrated area to obtain

$$
\int d \Omega \int \boldsymbol{J} \cdot d \boldsymbol{S} \sim 2 \pi S \int \sin \alpha \cos \alpha J(\alpha, E) d \alpha,
$$

where $\alpha$ is the pitch angle. We project the area $S$ along the magnetic field onto the magnetopause to obtain $S=S^{\prime} \cos \chi$, where $S^{\prime}$ means the projected area and $\chi$ indicates the angle between the magnetic field and the vector normal to the magnetopause. Then, we obtain

$\lambda \sim 2 \pi S^{\prime} \cos \chi \int d \alpha \sin \alpha \cos \alpha \int J(\alpha, E) d E$.

We integrate $J$ over the observed pitch angle and the measurement energy range $(187-3565 \mathrm{keV})$ to obtain

$$
\begin{aligned}
F & \equiv 2 \pi \int d \alpha \sin \alpha \cos \alpha \int J(\alpha, E) d E \\
& \sim 6 \times 10^{4} \mathrm{~cm}^{-2} \mathrm{~s}^{-1} .
\end{aligned}
$$

In order to discuss the order of $\lambda$, let us roughly assume $\cos \chi \sim 0.1$ and $S^{\prime} \sim 1 R_{E}^{2}$. Then we obtain $\lambda \sim 3 \times 10^{21} \mathrm{~s}^{-1}$. This value is much smaller than the oxygen ion loss rates across the magnetopause estimated by Seki et al. (2001) and Zong et al. (2001): $\sim 10^{23}$ to $10^{24} \mathrm{~s}^{-1}$ (the former and the latter assumed the open areas of $\sim 30 R_{E}^{2}$ and $5 R_{E}^{2}$, respectively). However, our integration is limited to the high energies $(>180 \mathrm{keV})$, hence the estimation is rather low. Instead, if we use the obtained moments $n \sim 2 \mathrm{cc}^{-1}$ as total density of oxygen ions and protons in the magnetosphere (11:2011:29 UT), the number density ratio $\mathrm{O}^{+} / \mathrm{H}^{+}$of 0.15 , and $v_{\|} \sim 500 \mathrm{~km} / \mathrm{s}$, we acquire the number flux of

$$
\begin{aligned}
F^{\prime} & \equiv n v_{\|} \\
& \sim 1.5 \times 10^{7} \mathrm{~cm}^{-2} \mathrm{~s}^{-1} .
\end{aligned}
$$

Then the loss rate is much larger: $\lambda \sim 6 \times 10^{23} \mathrm{~s}^{-1}$ (again we assumed $S^{\prime} \sim 1 R_{E}{ }^{2}, \cos \chi \sim 0.1$ ).

Since Seki et al. (2001) calculated the net escape of oxygen ions from the terrestrial magnetosphere as $\sim 5 \times 10^{24} \mathrm{~s}^{-1}$, the escape of the oxygen ions through magnetopause reconnection under northward IMF is possibly significant for the terrestrial atmospheric evolution, especially if the reconnection area is larger than a few $R_{E}{ }^{2}$.

Let us now discuss the $D_{s t}$ recovery. Note that the observed Sym- $H$ recovery, during the interval from 11:20 to $13: 00 \mathrm{UT}$, is $19 \mathrm{nT}$, and hence the recovery rate is $\sim 274 \mathrm{nT} /$ day. In order to estimate the influence of the energetic oxygen outflow on the $D_{s t}(\mathrm{Sym}-H)$ recovery, we use the Dessler-Parker-Sckopke relation (Dessler and Parker, 1959; Sckopke, 1966):

$$
\frac{\Delta B_{z}}{B_{s}}=-\frac{2 E}{3 E_{M}},
$$

where $\Delta B_{z}$ and $B_{s}$ are the variation of the magnetic field at the centre of the Earth caused by the ring current, and the magnetic field intensity at the surface of the Earth, respectively. $E$ and $E_{M}$ are the ring current total energy and the magnetic energy of the Earth, respectively. By using $B_{s} \sim 3 \times 10^{4} \mathrm{nT}$ and $E_{M} \sim 5 \times 10^{33} \mathrm{keV}$ for the Earth, we obtain

$\Delta B_{z} \sim-4 \times 10^{-30} E$,

where $\Delta B_{z}$ and $E$ are in the units of $\mathrm{nT}$ and $\mathrm{keV}$, respectively. Then we differentiate the above equation with respect to time, to obtain

$\frac{d \Delta B_{z}}{d t} \sim-4 \times 10^{-30} \frac{d E}{d t}$.

From the above equation, the influence of the oxygen ion escape on the $D_{s t}$ recovery can be estimated as:

$$
\left(\frac{d D_{s t}}{d t}\right)_{\text {o.e. }} \sim 4 \times 10^{-30} E_{0} \lambda,
$$

where $\left(d D_{s t} / d t\right)_{\text {o.e. }}$ denotes the contribution of the oxygen ion escape to the $D_{s t}$ recovery, and $E_{0}$ is the characteristic energy of the ring current oxygen ions $(\sim 50 \mathrm{keV})$. If we use $\lambda$ integrated above $\sim 180 \mathrm{keV}$, we obtain $\left(d D_{s t} / d t\right)_{o . e .} \sim 0.04 \mathrm{nT} /$ day, which is rather negligible. If, instead, we take $\lambda \sim 6 \times 10^{23} \mathrm{~s}^{-1}$, the recovery rate is $\left(d D_{s t} / d t\right)_{\text {o.e. }} \sim 11 \mathrm{nT} /$ day. Note that the calculated value is for the unit open area $\left(1 R_{E}^{2}\right)$. If the area of the ion escape is larger, e.g. $20 R_{E}{ }^{2}$, the recovery rate is as high as $220 \mathrm{nT} /$ day, and this loss process can contribute to the total $D_{s t}$ recovery significantly.

The above results are summarised in Table 1. Note that the loss rates and $\left(d D_{s t} / d t\right)_{\text {o.e. }}$ are normalised by the unit open area (i.e. $1 R_{E}{ }^{2}$ ) on the magnetopause.

More accurate estimation of the escape rate and the contribution to the $D_{s t}$ recovery requires the determination of 
the open area by a constellation of low-altitude satellites and/or ground observations, and the determination of reliable particle flux by measurements of medium-energy ions (10-200 keV/q) around the magnetopause in future missions (e.g. Kasahara et al., 2008).

\section{Summary}

We examined the dayside magnetopause skimming by Geotail around the equatorial plane, during a storm recovery phase on 15 May 2005. We focused on the time period from 11:20 UT to 13:00 UT, in which Geotail observed highenergy ( $>180 \mathrm{keV}$ ) oxygen ions escaping antiparallel to the magnetic field in the near-equatorial magnetosheath. In this time period, the IMF was constantly northward and the magnetic reconnection occurrence northward and duskward of Geotail is indicated by the Walén analysis and the ion bulk flows in the boundary layer. Low-energy $(\sim \mathrm{keV})$ particle precipitation in the polar cap observed by DMSP indicates that the closed magnetic field reconnected with the northward IMF. Thus, we conclude that the source of the observed oxygen ions is the ring current. This result suggests a new route for oxygen ion escape during northward IMF periods.

Acknowledgements. We thank the Geotail team for the precious data. We are grateful especially to R. W. McEntire and A. T. Y. Lui for providing the EPIC data. We also appreciate M. Nosé and Y. Ono for the data processing of EPIC data. The DMSP particle detectors were designed by Dave Hardy of AFRL, and data obtained from JHU/APL. We thank Dave Hardy, Gordon Wilson, and Patrick Newell for its use. We gratefully acknowledge the Center for Space Sciences at the University of Texas at Dallas and the US Air Force for providing the DMSP thermal plasma data. Valuable comments by one referee are highly appreciated. S. Kasahara was supported by a JSPS Research Fellowship for Young Scientists 19. 1222 .

Topical Editor I. A. Daglis thanks two anonymous referees for their help in evaluating this paper.

\section{References}

Abe, T., Whalen, B. A., Yau, A. W., Horita, R. E., Watanabe, S., and Sagawa, E.: EXOS D (Akebono) Suprathermal Mass Spectrometer Observations of the Polar Wind, J. Geophys. Res., 98(A7), 11 191-11 203, 1993.

André, M. and Yau, A. W.: Theories and observations of ion energization and outflow in the high latitude magnetosphere, Space Sci. Rev., 80, 27-48, 1997.

Cowley, S. W. H.: The cause of convection in the earth's magnetosphere - A review of developments during the IMS, Rev. Geophys., 20, 531-565, 1982.

Cowley, S. W. H.: Theoretical Perspectives of the Magnetopause: A Tutorial Review, Physics of the Magnetopause; Geophysical Monograph 90, edited by: Song, P., Sonnerup, B. U. Ö., and Thomsen, M. F., Published by the American Geophysical Union, Washington, D.C., p. 29-43, 1995.
Daglis, I. A., Axford, W. I., Sarris, E. T., Livi, S., and Wilken, B.: Particle Acceleration in Geospace and its Association with Solar Events, Solar Phys., 172, 287-296, 1997.

Daglis, I. A., Kozyra, J. U., Kamide, Y., Vassiliadis, D., Sharma, A. S., Liemohn, M. W., Gonzalez, W. D., Tsurutani, B. T., and Lu, G.: Intense space storms: Critical issues and open disputes, J. Geophys. Res., 108(A5), 1208, doi:10.1029/2002JA009722, 2003.

Daly, P. W., Saunders, M. A., Rijnbeek, R. P., Sckopke, N., and Russell, C. T.: The Distribution of Reconnection Geometry in Flux Transfer Events Using Energetic Ion, Plasma and Magnetic Data, J. Geophys. Res., 89(A6), 3843-3854, 1984.

Dessler, A. J. and Parker, E. N.: Hydromagnetic Theory of Geomagnetic Storms, J. Geophys. Res., 64, 2239-2252, 1959.

Ebihara, Y., Yamada, M., Watanabe, S., and Ejiri, M.: Fate of outflowing suprathermal oxygen ions that originate in the polar ionosphere, J. Geophys. Res., 111, A04219, doi:10.1029/2005JA011403, 2006.

Farrugia, C. J., Sandholt, P. E., Denig, W. F., and Torbert, R. B.: Observation of a correspondence between poleward moving auroral forms and stepped cusp ion precipitation, J. Geophys. Res, 103, 9309-9316, 1998.

Gosling, J. T., Thomsen, M. F., Bame, S. J., Elphic, R. C., and Russell, C. T.: Plasma Flow Reversals at the Dayside Magnetopause and the Origin of Asymmetric Polar Cap Convection, J. Geophys. Res., 95(A6), 8073-8084, 1990.

Greenspan, M. E., Anderson, P. B., and Pelagatti, J. M.: Characteristics of the thermal plasma monitor (SSIES) for the Defense Meteorological Satellite Program (DMSP) spacecraft S8 through S10, Rep. AFGLTR-86-0227, 1986.

Hamilton, D. C., Gloeckler, G., Ipavich, F. M., Stüdemann, W., Wilken, B., and Kremser, G.: Ring Current Development During the Great Geomagnetic Storm of February 1986, J. Geophys. Res., 93(A12), 14343-14 355, 1988.

Hanson, W. B., Heelis, R. A., Power, R. A., Lippincott, C. R., Zuccaro, D. R., Holt, B. J., Harmon, L. H., and Sanatani, S., The Retarding Potential Analyzer for Dynamics Explorer-B, Space Sci. Instrum., 5, 503-510, 1981.

Hardy, D. A., Schmitt, L. K., Gussenhoven, M. S., Marshall, F. J., Yeh, H. C., Schumaker, T. L., Hube, A., and Pantazis, J.: Precipitating electron and ion detectors (SSJ/4) for the block 5D/flights 6-10 DMSP satellites: Calibration and data presentation, Rep. AFGL-TR-84-0317, Air Force Geophys. Lab., Hanscom Air Force Base, Mass, 1984.

Heelis, R. A., Hanson, W. B., Lippincott, C. R., Zuccaro, D. R., Harmon, L. H., Holt, B. J., Doherty, J. E., and Power, R. A.: The Ion Drift Meter for Dynamics Explorer-B., Space Sci. Instrum., 5, 511-521, 1981.

Kasahara, S., Asamura, K., Ogasawara, K., Mitani, T., Hirahara, M., Takashima, T., Saito, Y., and Mukai, T.: Medium Energy Ion Mass Spectrometer Capable of Measurements of Three Dimensional Distribution Functions in Space, IEEE Trans. Plasma Sci., 36, 841-847, doi:10.1109/TPS.2008.922500, 2008.

Keika, K., Nosé, M., Christon, S. P., and McEntire, R. W.: Acceleration sites of energetic ions upstream of the Earth's bow shock and in the magnetosheath: Statistical study on charge states of heavy ions, J. Geophys. Res., 109, A11104, doi:10.1029/2003JA009953, 2004.

Keika, K., Nosé, M., Ohtani, S., Takahashi, K., Christon, S. 
P., and McEntire R. W.: Outflow of energetic ions from the magnetosphere and its contribution to the decay of the storm time ring current, J. Geophys. Res., 110, A09210, doi:10.1029/2004JA010970, 2005.

Keika, K., Nosé, M., Brandt, P. C., Ohtani, S., Mitchell, D. G., and Roelof, E. C.: Contribution of charge exchange loss to the storm time ring current decay: IMAGE/HENA observations, J. Geophys. Res., 111, A11S12, doi:10.1029/2006JA011789, 2006.

Kokubun, S., Yamamoto, T., Acuna, M. H., Hayashi, K., Shiokawa, K., and Kawano, H.: The Geotail Magnetic Field Experiment, J. Geomag. Geoelectr., 46, 7-21, 1994.

Kozyra, J. U. and Liemohn, M. W.: Ring current energy input and decay, Space Sci. Rev., 109, 105-131, 2003.

Kozyra, J. U., Liemohn, M. W., Clauer, C. R., Ridley, A. J., Thomsen, M. F., Borovsky, J. E., Roeder, J. L., Jordanova, V. K., and Gonzalez, W. D.: Multistep Dst development and ring current composition changes during the 4-6 June 1991 magnetic storm, J. Geophys. Res., 107(A8), 1224, doi:10.1029/2001JA000023, 2002.

Marcucci, M. F., Bavassano Cattaneo, M. B., Pallocchia, G., et al.: Energetic magnetospheric oxygen in the magnetosheath and its response to IMF orientation: Cluster observations, J. Geophys. Res., 109, A07203, doi:10.1029/2003JA010312, 2004.

McComas, D. J., Bame, S. J., Barker, P., Feldman, W. C., Phillips, J. L., Riley, P., and Griffee, J. W.: Solar Wind Electron Proton Alpha Monitor (SWEPAM) for the Advanced Composition Explorer, Space Sci. Rev., 86, 563-612, 1998.

McEntire, R. W., Lui, A. T. Y., Krimigis, S. M., and Keath, E. P.: AMPTE/CCE Energetic Particle Composition Measurements During the September 4, 1984 Magnetic Storm, Geophys. Res. Lett., 12(5), 317-320, 1985.

Moore, T. E., Fok, M.-C., and Chandler, M. O.: The dayside reconnection X line, J. Geophys. Res. (Space Physics), 107, 1332, doi:10.1029/2002JA009381, 2002.

Mukai, T., Machida, S., Saito, Y., Hirahara, M., Terasawa, T., Kaya, N., Obara, T., Ejiri, M., and Nishida, A.: The Low Energy Particle (LEP) Experiment onboard the GEOTAIL Satellite, J. Geomag. Geoelectr., 46, 669-692, 1994.

Newell, P. T., Meng, C.-I., Sanchez, E. R., Burke, W. J., and Greenspan, M. E., Identification and observations of the plasma mantle at low altitude, J. Geophys. Res., 96, 35-45, 1991a.

Newell, P. T., Sanchez, E. R., Meng, C.-I., Burke, W. J., Greenspan, M. E., and Clauer, C. R.: The low-latitude boundary layer and the boundary plasma sheet at low altitude - Prenoon precipitation regions and convection reversal boundaries, J. Geophys. Res., 96, $21013-21023,1991 b$.

Nilsson, H., Waara, M., Arvelius, S., Marghitu, O., Bouhram, M., Hobara, Y., Yamauchi, M., Lundin, R., Rème, H., Sauvaud, J. A., Dandouras, I., Balogh, A., Kistler, L. M., Klecker, B., Carlson, C. W., Bavassano-Cattaneo, M. B., and Korth, A.: Characteristics of high altitude oxygen ion energization and outflow as observed by Cluster: a statistical study, Ann. Geophys., 24, 1099-1112, 2006, http://www.ann-geophys.net/24/1099/2006/.

Onsager, T. G., Scudder, J. D., Lockwood, M., and Russell, C. T.: Reconnection at the high-latitude magnetopause during northward interplanetary magnetic field conditions, J. Geophys. Res., 106, 25 467-25 488, 2001.

Paschalidis, N. P., Sarris, E. T., Krimigis, S. M., McEntire, R. W., Levine, M. D., Daglis, I. A., and Anagnostopoulos, G. C., Ener- getic ion distributions on both sides of the Earth's magnetopause, J. Geophys. Res., 99, 8687-8703, 1994.

Paschmann, G., Papamastorakis, I., Baumjohann, W., Sckopke, N., Carlson, C. W., Sonnerup, B. U. Ö., and Lühr, H.: The Magnetopause for Large Magnetic Shear: AMPTE/IRM Observations, J. Geophys. Res., 91(A10), 11 099-11 115, 1986.

Pitout, F., Newell, P. T., and Buchert, S. C.: Simultaneous high- and low-latitude reconnection: ESR and DMSP observations, Ann. Geophys., 20, 1311-1320, 2002,

http://www.ann-geophys.net/20/1311/2002/.

Posner, A., Schwadron, N. A., Zurbuchen, T. H., Kozyra, J. U., Liemohn, M. W., and Gloeckler, G.: Association of LowCharge-State Heavy Ions up to 200 Re upstream of the Earth's bow shock with geomagnetic disturbances, Geophys. Res. Lett., 29(7), 1099, doi:10.1029/2001GL013449, 2002.

Sckopke, N.: A General Relation between the Energy of Trapped Particles and the Disturbance Field near the Earth, J. Geophys. Res, 71, 3125-3130, 1966.

Seki, K., Elphic, R. C., Hirahara, M., Terasawa, T., and Mukai, T.: On Atmospheric Loss of Oxygen Ions from Earth Through Magnetospheric Processes, Science, 291, 1939-1941, 2001.

Sibeck, D. G., McEntire, R. W., Lui, A. T. Y., Lopez, R. E., and Krimigis, S. M.: Energetic magnetospheric ions at the dayside magnetopause - Leakage or merging?, J. Geophys. Res., 92, 12 097-12 114, 1987.

Smith, C. W., L'Heureux, J., Ness, N. F., Acuña, M. H., Burlaga, L. F., and Scheifele, J.: The ACE Magnetic Fields Experiment, Space Sci. Rev., 86, 612-632, 1998.

Sonnerup, B. U. O., Paschmann, G., Papamastorakis, I., Sckopke, N., Haerendel, G., Bame, S. J., Asbridge, J. R., Gosling, J. T., and Russell, C. T.: Evidence for magnetic field reconnection at the earth's magnetopause, J. Geophys. Res., 86, 10 049-10067, 1981.

Sonnerup, B. U. O., Papamastorakis, I., Paschmann, G., and Luhr, H.: Magnetopause properties from AMPTE/IRM observations of the convection electric field: Method development, J. Geophys. Res., 92, 12 137-12 159, 1987.

Sonnerup, B. U. Ö., Paschmann, G., and Phan, T. D.: Fluid Aspects of Reconnection at the Magnetopause: In Situ Observations, edited by: Song, P., Sonnerup, B. U. Ö., and Thomsen, M. F., Physics of the Magnetopause, AGU Monograph 90, Washington, D.C., pp. 93-100, 1995.

Speiser, T. W., Williams, D. J., and Garcia, H. A.: Magnetospherically Trapped Ions as a Source of Magnetosheath Energetic Ions, J. Geophys. Res., 86(A2), 723-732, 1981.

Tsyganenko, N. A. and Stern, D. P.: Modeling the global magnetic field the large-scale Birkeland current systems, J. Geophys. Res. 101, 27 187-27 198, 1996.

Williams, D. J., McEntire, R. W., Schlemm II, C., Lui, A. T. Y., Gloeckler, G., Christon, S. P., and Gliem, F.: GEOTAIL Energetic Particles and Ion Composition Instrument, J. Geomag. Geoelectr., 46, 39-57, 1994.

Winglee, R. M., Skoug, R. M., McCarthy, M. P., et al.: Modeling of Upstream Energetic Particle Events Observed by WIND, Geophys. Res. Lett., 23(10), 1227-1230, 1996.

Zong, Q.-G., Wilken, B., Fu, S. Y., Fritz, T. A., Korth, A., Hasebe, N., Williams, D. J., and Pu, Z. Y.: Ring current oxygen ions escaping into the magnetosheath, J. Geophys. Res., 106, $25541-$ $25556,2001$. 